\title{
Computed tomography-guided trans-pulmonary-hepatic approach coil localization for pulmonary nodules near the right lung base
}

\author{
Ya-Ge Zheng', Tao Wang ${ }^{2}$, Pan-Hao Rong ${ }^{2}$, Yu-Fei Fu ${ }^{2}$ \\ ${ }^{1}$ Department of Interventional Radiology, Xuzhou City Hospital of Traditional Chinese Medicine, Xuzhou, China \\ 2Department of Radiology, Xuzhou Central Hospital, Xuzhou, China
}

Kardiochirurgia i Torakochirurgia Polska 2021; 18 (3): 173-176

\begin{abstract}
Introduction: Video-assisted thoracoscopic surgery (VATS)-guided diagnostic wedge resection (WR) is frequently used for removal of pulmonary nodules (PNs). Preoperative computed tomography (CT)-guided coil localization (CL) is often used for guidance. Aim: To evaluate the feasibility, safety, and effectiveness of CT-guided trans-pulmonary-hepatic approach CL of PNs near the right lung base.

Material and methods: Consecutive patients with PNs who underwent CT-guided CL followed by VATS-guided WR at our center from January 2018 to December 2020 were analyzed.

Results: One hundred and twenty-two patients with PNs underwent CT-guided CL followed by VATS-guided WR at our center. Of them, 5 (4.1\%) patients had PNs near the right lung base and underwent the CT-guided trans-pulmonary-hepatic approach $\mathrm{CL}$ procedures. The technical success rate of CL was $100 \%$. The VATS-guided WR was $100 \%$ successful. No patient required thoracotomy.
\end{abstract}

Conclusions: CT-guided trans-pulmonary-hepatic approach CL for PNs located near the right lung base is technically feasible.

Key words: localization, trans-pulmonary-hepatic approach, coil, pulmonary nodule.

\section{Introduction}

Video-assisted thoracoscopic surgery (VATS)-guided diagnostic wedge resection (WR) is frequently used for the removal of pulmonary nodules (PNs) [1-3]. To increase its success rate, preoperative computed tomography (CT)-guided coil localization $(\mathrm{CL})$ is often used for guidance [1-3]. The use of preoperative $\mathrm{CL}$ assessment may also reduce the requirement for resection or thoracotomy on $\mathrm{PN}$ diagnosis [2].

With peripheral PNs, the coils are usually located at the peripheral pleura [1-3]. Recently, CL has been successfully used in difficult cases including multiple, sub-fissural, and scapula-blocked PNs [4-6]. Besides these, some patients have PNs near the base of the right lung. According to the principle of $\mathrm{CT}$-guided $\mathrm{CL}$, the coil should be localized at the pleura which is nearest to the target PN $[6,7]$. Therefore, when we encounter PNs near the base of the right lung, the coil should be localized at the right lung base pleura. Because the right lung base is close to the diaphragm and liver, in these cases, the coil should be placed in both the lung and liver to locate the PNs.

\section{Aim}

Here, we aimed to assess the feasibility, efficacy, and safety of preoperative CL using the trans-pulmonary-hepatic approach for PNs near the right lung base.

\section{Material and methods}

This retrospective single-center study was approved by our Institutional Review Board. As the study was retrospective, the patient informed consent requirement was waived.

\section{Study design}

One hundred and twenty-two patients with PNs underwent CT-guided CL followed by VATS-guided WR at our center between January 2018 and December 2020. Of these, 5 (4.1\%) patients had PNs near the right lung base and underwent CT-guided trans-pulmonary-hepatic approach $\mathrm{CL}$ procedures. The resection approach was individualized for each patient, decided by discussion between radiologists, oncologists, and thoracic surgeons.

Address for correspondence: Yu-Fei Fu MD, Department of Radiology, Xuzhou Central Hospital, Xuzhou, China, phone: +86 18112000304 , e-mail: fuyufei1985@163.com

Received: 6.05.2021, accepted: 4.07.2021. 


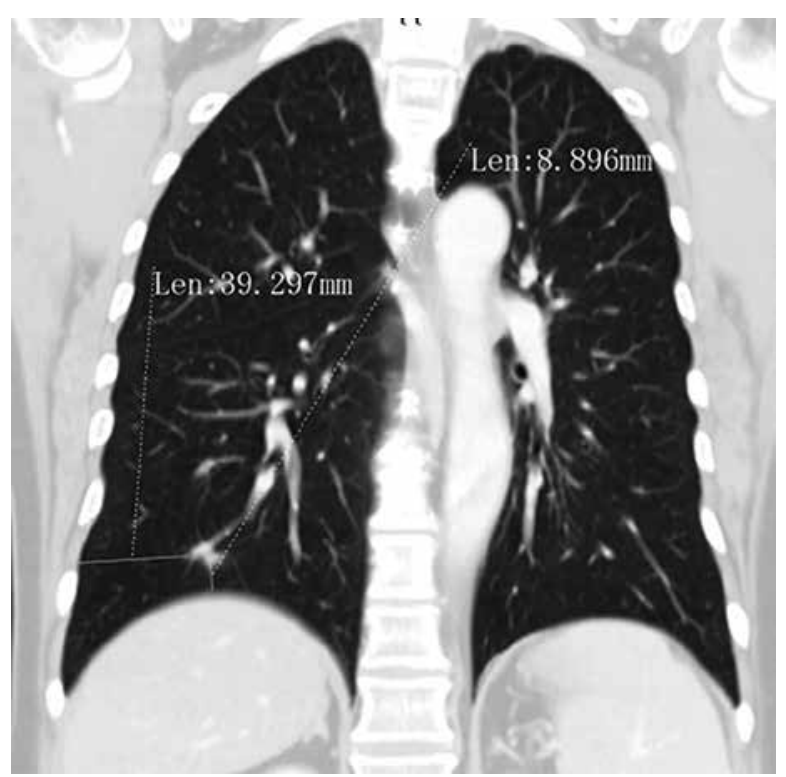

Figure 1. A PN near the lung base that is suitable for trans-pulmo nary-hepatic approach CL

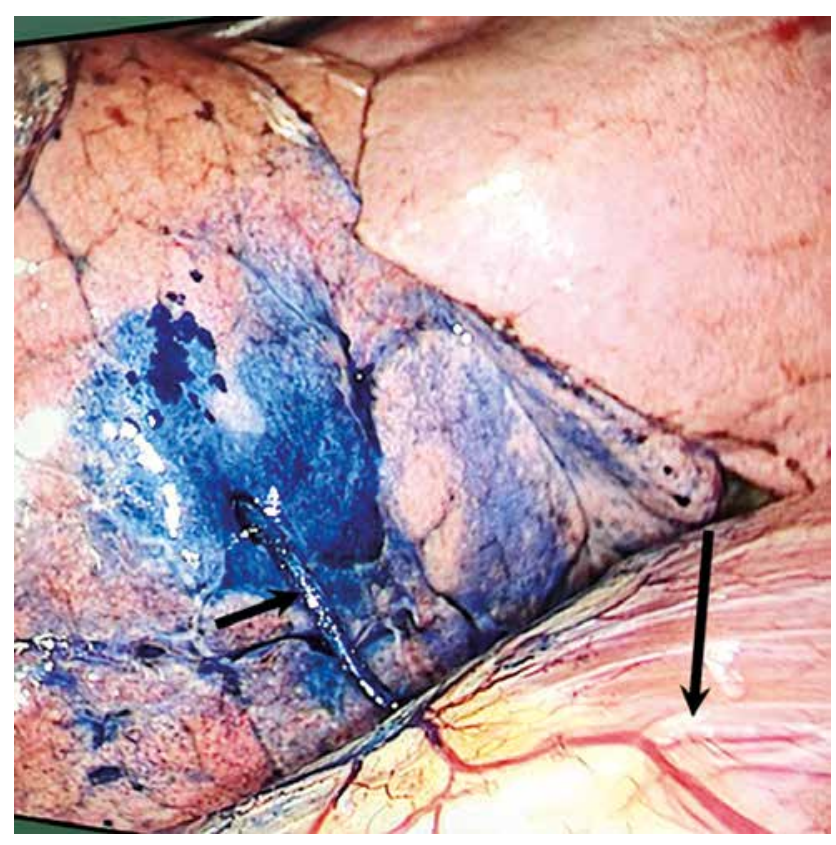

Figure 3. The coil (short arrow) partially inserted into the lung indicating successful CL. The long arrow indicates the diaphragm

The inclusion criteria were: (a) a CT-based maximal longaxis PN diameter $\leq 30 \mathrm{~mm}$; (b) a PN located in the right lower lobe; (c) a PN-peripheral pleura distance $>30 \mathrm{~mm}$; and (d) a PN-lung base distance $\leq 20 \mathrm{~mm}$ (Figure 1).

The exclusion criteria were: (a) a benign $\mathrm{PN}$, characterized by typical appearance, calcifications, and nodules that showed reduced size on follow-up; (b) a PN diameter $<5 \mathrm{~mm}$; and (c) evidence of active infection or bleeding, as well as abnormal coagulation and reduced cardiopulmonary reserve.

CT-guided trans-pulmonary-hepatic approach CL was performed under local anesthesia by a chest radiologist with experience (over 10 years) in CT-guided procedures.
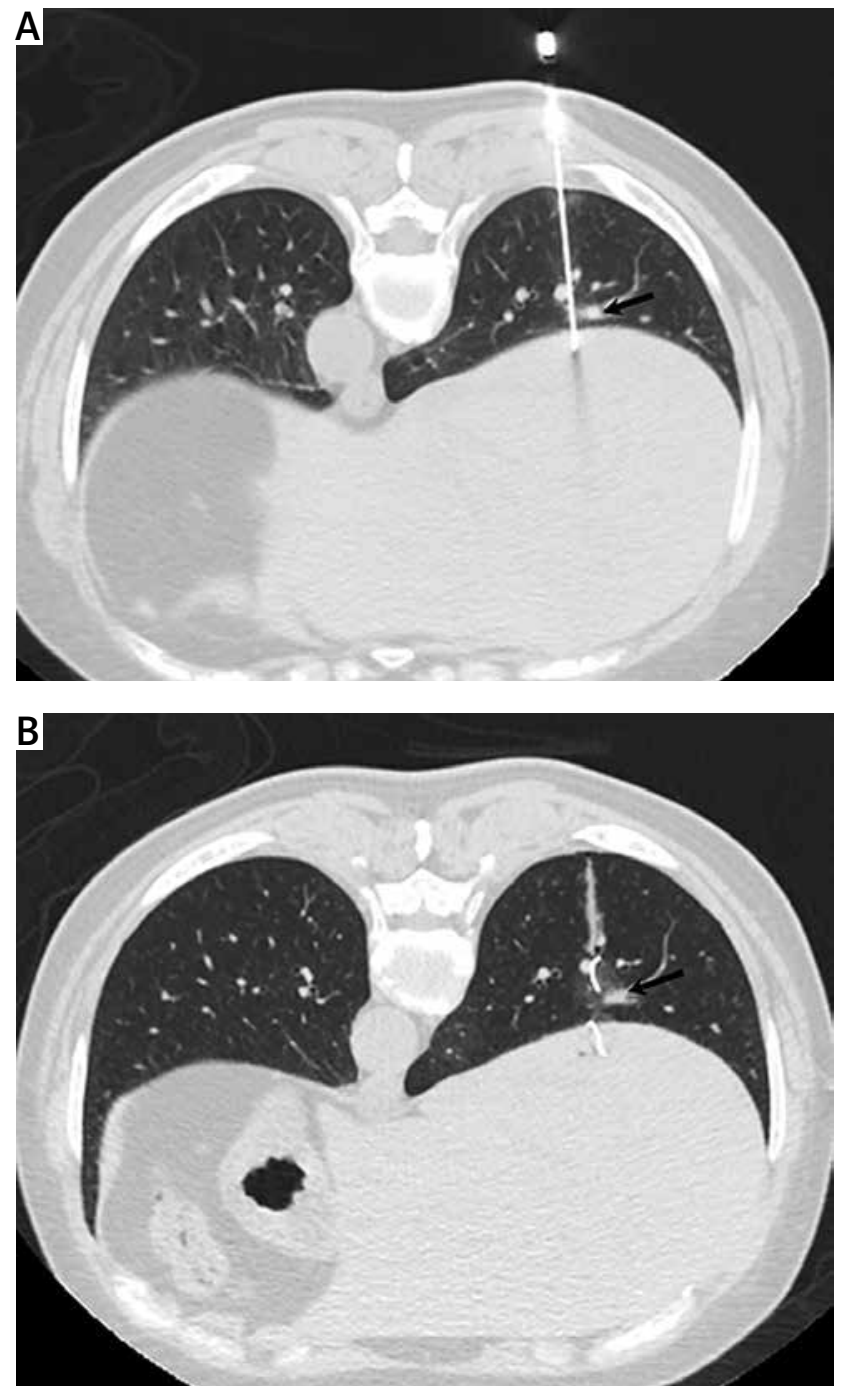

Figure 2. A - Insertion of the needle (18G) into the lung and liver. The needle body is near the PN (arrow). B - The coil is positioned near the PN (arrow) and inserted into the lung and liver

During the procedure, patients were required to avoid moving and to breathe regularly.

The optimal pathway for the needle was determined by preoperative CT. The needle (18G, Precisa, Roma, Italy) was inserted into the lung and liver (Figure $2 \mathrm{~A}$ ). The length of the intra-hepatic needle path was controlled within $10 \mathrm{~mm}$ while that in the lung was within $10 \mathrm{~mm}$ of the PN. Then, a 0.038-inch diameter and $50 \mathrm{~mm}$ long coil (Cook, IN, USA) was partly inserted into the liver. The needle was then removed smoothly so that the residual coil was inserted in the lung near the PN (Figure 2 B). When the coil localization was completed, we inserted the needle again and injected $\sim 0.5 \mathrm{ml}$ of methylene-blue for additional localization.

The patient then received a postoperative CT scan to determine the likelihood of procedure-related complications.

VATS-guided WR was performed within 24 hours of $\mathrm{CL}$. The WR procedure was conducted according to the visualization of the coil (Figure 3) using a cutting boundary $>2 \mathrm{~cm}$ from the coil. The coil was also removed from the liver during the VATS procedures. 
The resected lung tissue was rapidly assessed by our Department of Pathology and if the PN was considered to have progressed beyond the mini-invasive adenocarcinoma (MIA) stage, further lymph node dissection or lobectomy was performed.

\section{Definitions}

CT-guided trans-pulmonary-hepatic approach $\mathrm{CL}$ was considered technically successful when partial insertion of the coil into the lung was observed during the VATS procedure. Wedge resection was considered technically successful when the PN could be found in the resected lung parenchymal tissue.

\section{Results}

\section{Patients}

Table I shows the baseline data of the five patients (male: 3; female: 2). No patient had a history of malignancy. Each patient had one PN near the right lung base and no other PNs were found among any of these 5 patients. The median age, PN diameter, lesion-lung base distance, and lesion-lateral pleura distance were 58 years (range: 52-65 years), $8 \mathrm{~mm}$ (range: $5-9 \mathrm{~mm}$ ), $6 \mathrm{~mm}$ (range: $3-8 \mathrm{~mm}$ ), and $44 \mathrm{~mm}$ (range: $39-61 \mathrm{~mm}$ ), respectively.

\section{Technical success of $C L$}

The technical success rate of CT-guided trans-pulmonary-hepatic approach CL was $100 \%$ (Table II). Patients were placed in the prone position and each PN was localized with one coil and the median distance of the intrapulmonary needle path was $55 \mathrm{~mm}$ (range: 50-69 mm). The median CL duration was 15 min (range: 12-16 min).

Intrapulmonary bleeding around the pathway was observed in all patients. Only one patient (patient 3) experienced asymptomatic pneumothorax after CL. The pneumo- thorax was detected by the postoperative CT scan. None of the CL-related complications influenced VATS. No patient experienced any complications in liver parenchyma.

\section{Technical success of WR}

The VATS-guided WR was 100\% successful. The coil remained in position in all patients and no patient required thoracotomy. The median WR procedure duration was 35 min (range: 30-40 min). The pathological diagnoses of these five PNs were invasive adenocarcinoma $(n=1)$, minimally invasive adenocarcinoma $(n=2)$, adenocarcinoma in situ $(n=1)$, and benign PN $(n=1)$. One patient (No. 1$)$ where the PN was diagnosed as invasive adenocarcinoma underwent additional lobectomy. All malignant PNs were "RO" resected. The median blood loss was $50 \mathrm{ml}$ (range: 20-100 ml).

\section{Discussion}

In this study, we illustrate our experience using preoperative trans-pulmonary-hepatic approach CL for PNs near the base of the right lung. Firstly, the technical success rate of $\mathrm{CL}$ in this study was $100 \%$, which agrees with previous reports of CT-guided CL for PNs (89.6-94.4\%) [1-3]. The high success rate of trans-pulmonary-hepatic approach $\mathrm{CL}$ can be attributed to the following factors: (a) the relative anatomical position and morphology of the right lung base and liver allow the puncture needle to puncture both the lung and liver simultaneously; (b) the coil is long enough $(50 \mathrm{~mm})$ to be positioned in the lung and liver simultaneously; and (c) the length of the coil in the liver was controlled within $10 \mathrm{~mm}$, allowing most of the coil to be inserted into the lung. This ensured the stable positioning of the coil within the lung.

Li et al. [5] and Xia et al. [7] performed CT-guided trans-fissural $\mathrm{CL}$ for patients with sub-fissural PNs with

Table I. Baseline data of the 5 patients

\begin{tabular}{cccccc} 
No. & $\begin{array}{c}\text { Age } \\
\text { [years]/gender }\end{array}$ & $\begin{array}{c}\text { Diameter } \\
{[\mathrm{mm}]}\end{array}$ & Nature & $\begin{array}{c}\text { Lesion-lung base distance } \\
{[\mathrm{mm}]}\end{array}$ & $\begin{array}{c}\text { Lesion-peripheral pleura distance } \\
{[\mathrm{mm}]}\end{array}$ \\
\hline 1 & $55 /$ female & 5 & Solid & 39 & 61 \\
\hline 2 & $52 /$ male & 5 & GGN & 5 & 4 \\
\hline 4 & $60 /$ female & 8 & Solid & 7 & 42 \\
\hline 5 & $65 /$ male & 9 & Mixed GGN & 6 & 52 \\
\hline
\end{tabular}

GGN - ground glass nodule.

Table II. Details of coil localization

\begin{tabular}{|c|c|c|c|c|c|}
\hline No. & $\begin{array}{l}\text { Technical } \\
\text { success }\end{array}$ & $\begin{array}{l}\text { Patients' } \\
\text { position }\end{array}$ & $\begin{array}{l}\text { Duration } \\
\text { [min] }\end{array}$ & $\begin{array}{c}\text { Intrapulmonary needle } \\
\text { path distance }[\mathrm{mm}]\end{array}$ & Complication \\
\hline 1 & Yes & Prone & 16 & 52 & Bleeding around the pathway \\
\hline 2 & Yes & Prone & 12 & 69 & Bleeding around the pathway \\
\hline 3 & Yes & Prone & 15 & 50 & Asymptomatic pneumothorax; Bleeding around the pathway \\
\hline 4 & Yes & Prone & 14 & 55 & Bleeding around the pathway \\
\hline 5 & Yes & Prone & 16 & 61 & Bleeding around the pathway \\
\hline
\end{tabular}


both teams achieving $100 \%$ technical success. There are certain similarities between the CT-guided trans-fissural and trans-pulmonary-hepatic approach CL procedures. For trans-fissural CL, the coil is localized on the pleural fissures between the target site and the adjacent lobes while for trans-pulmonary-hepatic approach CL, the coil is positioned on the lung base between the lung and the diaphragm.

VATS-guided WR was successful in all (100\%) of the patients in this study. This high success rate is comparable to those previously reported for CT-guided CL for $\mathrm{PN}$ resection (93.1-97.2\%) [1-3] and can be attributed to the success of the CL. In addition, the injection of methylene-blue enhanced the effectiveness of localization. Furthermore, no coils were dislodged from the lung during the VATS procedures in this study. Although the coil was placed into the lung and liver simultaneously, most of the coil was positioned in the lung. Thus, when the lung was collapsed during the VATS procedure, the coil remained in place.

The incidence of CL-related pneumothorax was $20 \%$ in this study. This is slightly higher than previously reported (9-15.8\%) for CT-guided CL for PN resection [1, 8, 9]. Furthermore, all patients experienced intrapulmonary bleeding around the path. These findings could be attributed to the long intrapulmonary distance of the needle path (median $55 \mathrm{~mm}$ ). For the more common PNs situated near the lateral pleura, the intrapulmonary needle path distance was usually less than $20 \mathrm{~mm}$ [1,9]. It is well known that a longer intrapulmonary needle path is associated with a higher complication rate [10]. Furthermore, we did not observe any complications in liver parenchyma. This finding might indicate that it was safe to place a partial coil in the liver parenchyma.

This study has several limitations. Firstly, it is a singlecenter retrospective analysis and, therefore, risks selection bias. Secondly, the small sample size necessitates further research to validate the findings. There was also no control group. However, given that CT-guided trans-pulmonary-hepatic approach $\mathrm{CL}$ is not a common procedure and is used only in limited numbers of patients, we consider that our findings nevertheless show the feasibility and safety of the procedure.

\section{Conclusions}

The preoperative CT-guided trans-pulmonary-hepatic approach CL for PNs located near the right lung base is technically feasible. Further studies with a large sample size are still needed.

\section{Disclosure}

The authors report no conflict of interest.

\section{References}

1. Fu YF, Zhang M, Wu WB, Wang T. Coil localization-guided video-assisted thoracoscopic surgery for lung nodules. J Laparoendosc Adv Surg Tech A 2018; 28: 292-297.

2. Głowacki J, Legaszewski T, Dzielicki J, et al. Computed tomography-guided hookwire localization of solid lung tumours before videothoracoscopic surgery. Kardiochir Torakochir Pol 2008; 5: 43-46.

3. Zhang ZD, Wang HL, Liu XY, Xia FF, Fu YF. Methylene blue versus coil-based computed tomography-guided localization of lung nodules. Thorac Cardiovasc Surg 2020; 68: 540-544.

4. Fu YF, Gao YG, Zhang M, Wang T, Shi YB, Huang YY. Computed tomographyguided simultaneous coil localization as a bridge to one-stage surgery for multiple lung nodules: a retrospective study. J Cardiothorac Surg 2019; 14: 43.

5. Li EL, Cao W, Li Y, Zhang M. Computed tomography-guided coil localization for sub-fissural lung nodules. Medicine 2020; 99: e22030.

6. Liu X, Cao W, Xu QS. Computed tomography-guided coil localization for scapula-blocked pulmonary nodules: a trans-scapular approach. Medicine 2021; 100: e24333.

7. Xia FF, Shi YB, Wang T, Fu YF. Computed tomography-guided transfissural coil localization of lung nodules. Thorac Cardiovasc Surg 2020; 68: 545-548.

8. Su TH, Fan YF, Jin L, He W, Hu LB. CT-guided localization of small pulmonary nodules using adjacent microcoil implantation prior to video-assisted thoracoscopic surgical resection. Eur Radiol 2015; 25: 2627-2633.

9. Yang ZJ, Liang YH, Li M, Fang P. Preoperative computed tomography-guided coil localization of lung nodules. Minim Invasive Ther Allied Technol 2020; 29: 28-34.

10. Li GC, Fu YF, Cao W, Shi YB, Wang T. Computed tomography-guided percutaneous cutting needle biopsy for small $(\leq 20 \mathrm{~mm})$ lung nodules. Medicine 2017; 96: e8703. 